\title{
Novel bootstrapped CMOS differential logic family for ultra-low voltage SoCs
}

\author{
Byung-Hwa Jung, Sung-Chan Kang, Jae-Hyuk Oh, Yoon-Suk Park, \\ Yong-Ki Kim, Yong-Gu Kang, Jong-Woo Kim, and Bai-Sun Kong ${ }^{\text {a) }}$ \\ School of Information and Communication Engineering, Sungkyunkwan University, \\ 300 Chunchun-dong, Jangan-gu, Suwon, Gyunggi-do, 440-746, Korea \\ a)bskong@skku.edu
}

Abstract: This paper describes novel bootstrapped CMOS logic family operating at ultra-low supply voltages. The proposed logic family provides better switching performance than conventional bootstrapped logic family by isolating the bootstrapping circuit from timing-critical signal paths. The logic family also minimizes area overhead due to the bootstrapping circuit by adapting a differential structure having a single bootstrap capacitor shared between complementary outputs. Multi-input XOR/XNOR gates and 64-bit adders were designed in $0.18 \mathrm{um}$ CMOS process as test vehicles for assessing the performance. Comparison results indicate that the power-delay product of the proposed logic family is improved by up to $67 \%$ compared to conventional differential logic circuits at the supply voltage ranging from $0.5 \mathrm{~V}$ to $0.8 \mathrm{~V}$.

Keywords: bootstrapping, low voltage, low power, CMOS logic family

Classification: Integrated circuits

\section{References}

[1] M. Pedram and J. M. Rabaey, Power-aware design methodologies: Kluwer Academic Publication, 2002.

[2] L. G. Heller, et al., "Cascode voltage switch logic: a differential CMOS logic family," in ISSCC Dig. Tech. Papers, pp. 16-17, Feb. 1984.

[3] R. H. Krambeck, C. M. Lee, and H. S. Law, "High-speed compact circuits with CMOS," IEEE J. Solid-State Circuits, vol. sc-17, no. 3, pp. 614-619, June 1982.

[4] J. H. Lou and J. B. Kuo, "A $1.5 \mathrm{~V}$ full-swing bootstrapped CMOS large capacitive-load driver circuit suitable for low-voltage CMOS VLSI," IEEE J. Solid-State Circuits, vol. 32, no. 1, pp. 119-121, Jan. 1997.

[5] J. H. Lou and J. B. Kuo, "A 1.5-V CMOS all-N-logic true-single-phase bootstrapped dynamic-logic circuit suitable for low supply voltage and high-speed pipelined system operation," IEEE Trans. Circuits Syst. II, vol. 46, pp. 628-631, May 1999. 


\section{Introduction}

It is well known that low-power design has become one of the prime issues in system-on-a-chip (SoC) design. One effective way of minimizing the power consumption of digital CMOS circuits is to scaling down the power supply voltage [1]. This is mainly because the switching power consumption, the dominant portion of the total power consumption in digital CMOS circuits, has a quadratic dependency on the supply voltage. A serious problem associated with this voltage scaling-driven power reduction approach is a severe degradation of switching speed of the circuit. The reason for this drawback comes from the fact that the threshold voltages of MOS devices cannot be scaled down by the same amount as the supply voltage. This in turn reduces the effective gate-source voltage of devices, resulting in slower switching speed. Hence, the speed performance of high-speed dynamic CMOS logic families such as differential cascode voltage switch (DCVS) logic [2] and domino CMOS logic [3] is severely degraded as the supply voltage approaches device thresholds.

To allow digital CMOS circuits to operate fast at scaled supply voltages, the bootstrapped CMOS large capacitive-load driver [4] was proposed. It can improve the switching speed of the driver at low supply voltages by allowing the voltage of internal nodes to be boosted beyond the supply rails. However, since this circuit was proposed for use as large capacitive load driver, logic functions cannot be efficiently embedded into the circuit. For fast logic operation at low supply voltages, CMOS bootstrapped dynamic logic (BDL) [5] shown in Fig. 1-(a) was proposed. But, the speed of this logic family was not so high since the latency of bulky bootstrapping circuit was added to the overall latency of the logic family. Moreover, logic composition of this logic family is severely constrained since it is configured as a singleended structure. Although differential BDL shown in Fig. 1-(b) can overcome this constraint, design overhead in terms of silicon area is critical since it must have duplicate bootstrapping circuits.

To overcome these problems and to further improve the switching performance, novel bootstrapped CMOS differential logic family is introduced in this paper. Section 2 describes the circuit structure and operation of the proposed logic style. In Section 3, comparison results for some test circuits are presented to assess the performance of the proposed logic family. Finally, we present the conclusion in Section 4.

\section{Circuit Structure and Operation}

Figure 1-(c) shows the schematic diagram of the proposed bootstrapped CMOS differential logic (BCDL). It consists of a precharged differential logic block, a pair of complementary output drivers, and a bootstrapping circuit. The precharged differential logic block is constructed with a differential logic tree, precharge transistors (MP1 and MP2), keeper transistors (MP3 and MP4), and a bottom transistor (MN1). The differential logic tree implements logic function, and is enabled by the bottom transistor. Clock signal 


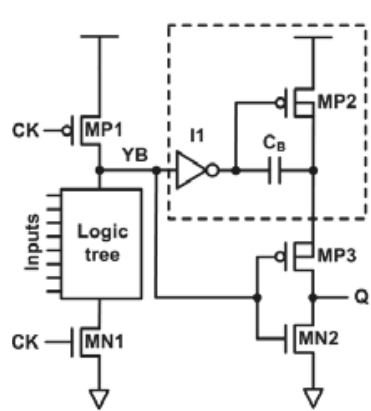

(a)

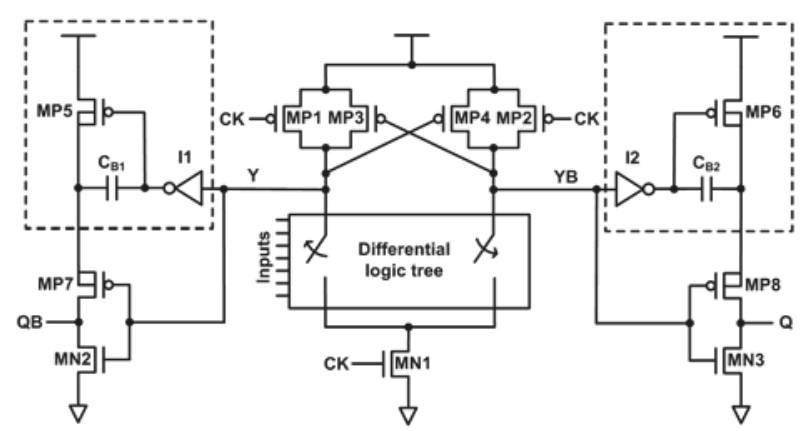

(b)

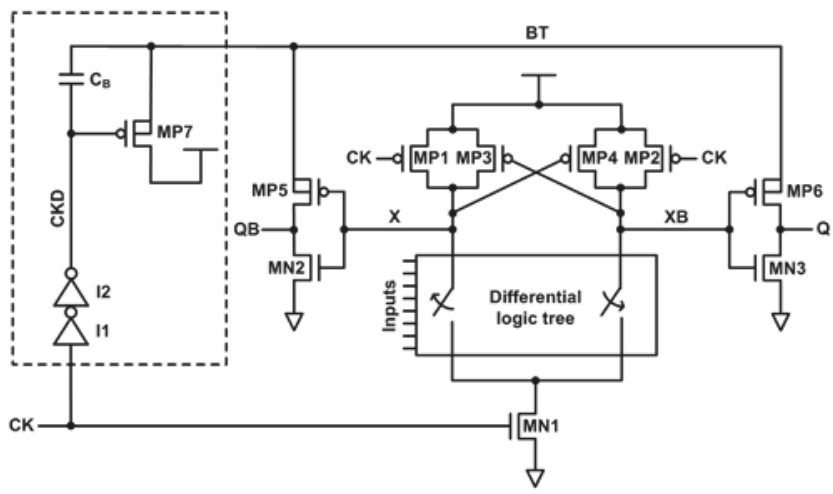

(c)

Fig. 1. Bootstrapped CMOS logic circuits: (a) CMOS bootstrapped dynamic logic (single-ended), (b) CMOS bootstrapped dynamic logic (differential), (c) proposed bootstrapped CMOS differential logic

$C K$ drives the precharge transistors and the bottom transistor to determine the phase of operation. Complementary outputs of the precharged differential logic block, $X$ and $X B$, are fed into the associated complementary output drivers in which transistor pairs, MP5-MN2 and MP6-MN3, drive complementary outputs, $Q B$ and $Q$, respectively. The left part of BCDL shown in dotted box, consisting of inverters I1, I2, transistor MP7, and bootstrap capacitor $\mathrm{C}_{\mathrm{B}}$, constitutes the bootstrapping circuit, and is used to boost the voltage of node $B T$ above the supply voltage. Note that the bodies of pchannel transistors, MP5, MP6, and MP7, whose source nodes are boosted above the supply voltage, are connected to the bootstrap node instead of VDD for avoiding any forward bias in source-substrate junctions.

BCDL has two phases of operation, the precharge phase and the bootstrapped evaluation phase. When $C K$ is low, the circuit is in the precharge phase. The differential logic tree is disabled during this phase since MN1 is fully off, and precharge nodes $X$ and $X B$ are precharged to the supply voltage by MP1 and MP2. Then, transistors MP5 and MP6 turn off while transistors MN2 and MN3 turn on, allowing complementary outputs $Q$ and $Q B$ to have low logic values. At the same time, since $C K D$ is low, MP7 turns on and a voltage equal to the supply voltage is applied to $C_{B}$. When 
$C K$ changes to high, the circuit goes into the bootstrapped evaluation phase. One of the precharge nodes is pulled down fast toward the ground by the differential logic tree during this phase according to the input data. (As seen below, the logic high of inputs has the boosted voltage level, so the speed of this pull-down is very fast.) Let us assume that inputs are such that $X B$ is evaluated low. MP6 and MN3 are then turned on and off, respectively, allowing $Q$ to be disconnected from the ground and connected to $B T$. At the same time, since $C K D$ transitions high, MP7 turns off, and the voltage of $B T$ are pulled up above VDD by capacitive coupling. This high voltage allows enhanced driving strength of MP6, resulting in a very fast pull-up of $Q$. Moreover, the bootstrapped voltage transferred to $Q$ also enhances the driving strength of the transistors in the differential logic tree of the next stage, further improving the switching performance of the logic family. When the circuit enters the precharge phase after $C K$ changes to low, $X B$ is precharged to the supply voltage, allowing $Q$ to return to low. When CKD transitions low, BT goes low. Note that the pull-down transition of the output during the precharge phase is not bootstrapped since this transition is not critical in terms of speed.

BCDL provides several important advantages as compared to conventional bootstrapped CMOS circuits. BCDL is better suited for logic composition than the bootstrapped CMOS driver since arbitrary logic functions can be efficiently embedded into the circuit. BCDL is also better than BDL in terms of switching speed, logical flexibility, and silicon area. As seen from Figs. 1-(a) and (b), each bootstrapping circuit (shown in each dotted box) in BDL is triggered by the pull-down transition of the associated precharge node, implying that the latency of the bootstrapping circuit will be included in the overall latency of the logic family. Since the latency of bootstrapping circuit is not negligible due to bulky bootstrap capacitor, this circuit structure makes the speed improvement of BDL not so much as expected by voltage bootstrapping. Moreover, the fact that the precharge node must drive the bootstrapping circuit also causes a significant capacitive burden to the node since the inverter (I1 in case of Fig. 1-(a)) to fast drive huge bootstrap capacitor should have a large size. The increase of parasitic capacitance on timing-critical nets such as precharge nodes inevitably results in further speed degradation of BDL. Meanwhile, in case of BCDL, the bootstrapping circuit is not triggered by the precharge node but by a delayed version of CK as shown in Fig. 1-(c). This allows the bootstrapping circuit to be completely isolated from the precharge node, giving no capacitive burden to the precharge node and no latency increase of the logic family. Hence, BCDL can provide far better switching performance than BDL at ultra-low supply voltages. Since the differential logic block and the bootstrapping circuit are connected in parallel, making the latency of the bootstrapping circuit identical to that of the differential logic block will result in an optimal switching performance. Another critical disadvantage of BDL shown in Fig. 1-(a) comes from that it is configured as a single-ended structure. With this structure, logic composition is severely constrained for implementing useful logic gates 
such as XOR and multiplexer requiring monotonically rising true and complement inputs. These complementary signals can be separately generated using two independent BDL gates having dual logic functions as shown in Fig. 1-(b), but this logic family can lead to increased silicon area due to the use of duplicate bootstrapping circuits. Meanwhile, BCDL is structured differentially causing no constraints on logic composition, and has a single bootstrapping circuit shared by complementary output drivers, achieving a minimum area overhead.

\section{Comparison and Discussion}

In order to assess the performance of the proposed logic family, XOR/XNOR gates and 64 -bit adders were designed in a $0.18 \mu \mathrm{m}$ CMOS process. The nominal threshold voltages of $\mathrm{p}$ - and n-channel transistors are $-0.44 \mathrm{~V}$ and $0.41 \mathrm{~V}$, respectively. The bootstrap capacitor for each bootstrapped logic family, whose capacitance value of $30 \mathrm{fF}$ was selected to achieve a boosted voltage of $200 \mathrm{mV}$, was implemented using an MOS transistor. The transistor widths of each gate were optimized individually to provide optimal performance. To achieve an optimized performance of BCDL, the latency of the bootstrapping circuit was made to be identical to that of the differential logic block by adjusting the size of inverters I1 and I2 in Fig. 1-(c). Each 64-bit adder whose block diagram is shown in Fig. 2 is composed of eight 8-bit adder sub-sections, and employ carry selection scheme to speed up the addition operation. For BDL and BCDL adders, a ripple carry chain was used in each 8-bit adder sub-section to fully exploit the speed enhancement by bootstrapping. For (non-bootstrapped) DCVS adder, Manchester carry chain was used for high-speed carry propagation. To compare the performance of these circuits, HSPICE simulation was performed including the parasitic components extracted from the layout. Fig. 3 compares the power-delay product (PDP) performance of XOR/XNOR gates designed with the conventional and proposed logic families. Fig. 3-(a) shows the performance depending on supply voltage change whose range is between $0.5 \mathrm{~V}$ and $0.8 \mathrm{~V}$. In case of DCVS gate, the performance is significantly degraded as the supply voltage decreases and approaches device thresholds. Although the performance

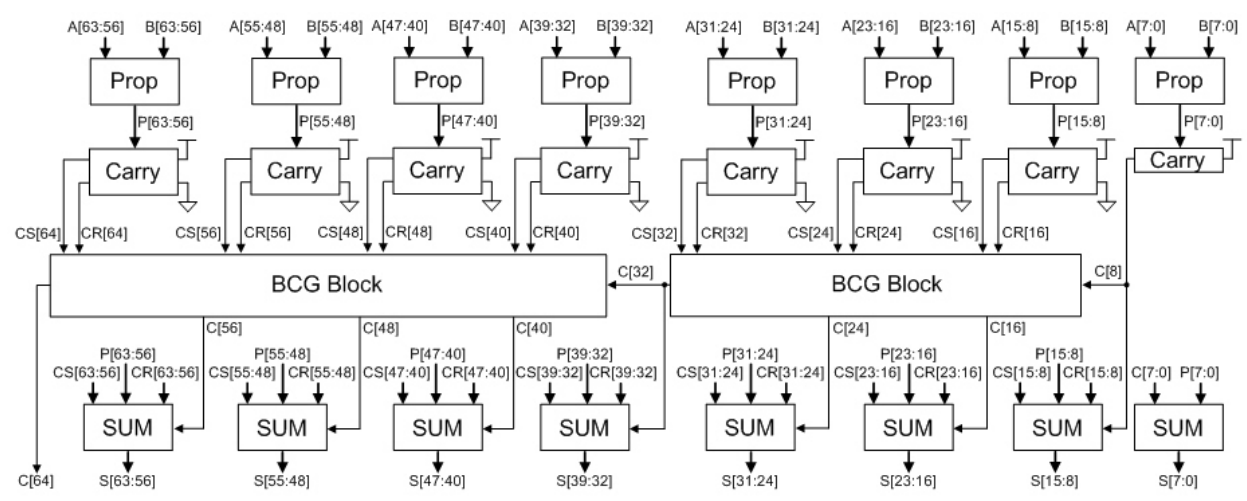

Fig. 2. The block diagram of 64-bit adder 


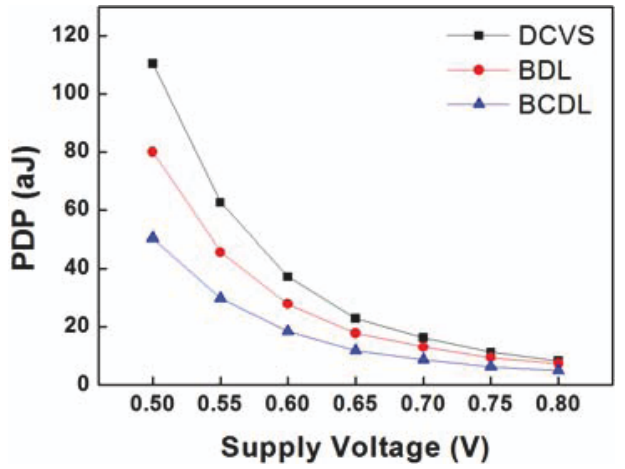

(a)

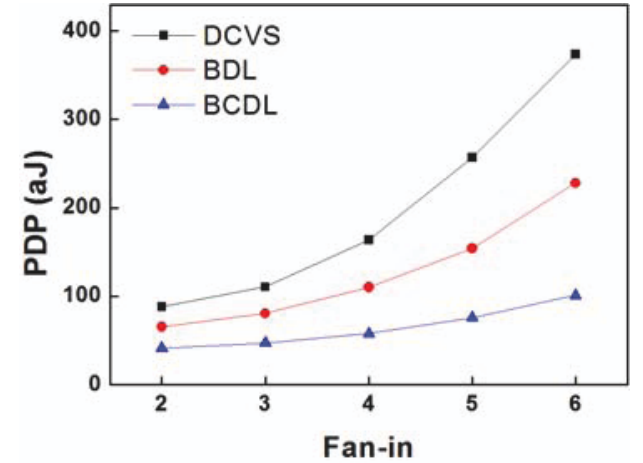

(b)

Fig. 3. Simulated comparison results $\mathrm{XOR} / \mathrm{XNOR}$ gates (supply voltage: $0.5 \mathrm{~V}$, temperature: $27^{\circ} \mathrm{C}$ ): (a) power-delay product versus supply voltage, (b) power-delay product versus fan-in number

of BDL gate is better than that of DCVS gate, its improvement was not enough because of latency increase due to the bootstrapping circuit located along the timing-critical path. Meanwhile, BCDL gate provides up to $55 \%$ and 37\% PDP improvements compared to DCVS and BDL gates, respectively. Fig. 3-(b) shows the PDP performance of the logic gates versus fan-in number where the number of fan-in ranges from 2 to 6 at a supply voltage of $0.5 \mathrm{~V}$. As shown in the figure, BCDL gates provide $53 \sim 73 \%$ improved PDP performance, indicating that BCDL maintains performance advantage for increased logic complexity. Table I summarizes the performance comparison of 64-bit adders. As shown in the table, BCDL adder occupies $17 \%$ less area than BDL adder due to reduced number of bootstrapping circuits. Although BCDL adder still has bootstrapping circuits, its area overhead to DCVS adder is less than 6\% due to significantly reduced logic tree size. (Since inputs to the logic tree have a boosted voltage, transistors in the logic tree have small widths.) Moreover, BCDL adder is much faster than DCVS and BDL adders, and thus, achieves $67 \%$ and $57 \%$ improvements in terms of PDP performance, respectively.

Table I. Simulated performance comparison for 64-bit adders (supply voltage: $0.5 \mathrm{~V}$, temperature: $\left.27^{\circ} \mathrm{C}\right)$

\begin{tabular}{c|c|c|c|c|c|c}
\hline & $\begin{array}{c}\text { Device } \\
\text { count }\end{array}$ & $\begin{array}{c}\text { Layout } \\
\text { Area }\left(\mu \mathrm{m}^{2}\right)\end{array}$ & $\begin{array}{c}\text { Latency } \\
(\mathrm{ns})\end{array}$ & $\begin{array}{c}\text { Power } \\
(\mathrm{uW})\end{array}$ & $\begin{array}{c}\text { PDP } \\
(\mathrm{fJ})\end{array}$ & Ratio \\
\hline DCVS & 5781 & 96914 & 102.4 & 5.1 & 522.2 & 1 \\
\hline BDL & 7911 & 122903 & 37.1 & 10.9 & 404.4 & 0.77 \\
\hline BCDL & 7536 & 102321 & 17.3 & 10.1 & 174.7 & 0.33 \\
\hline
\end{tabular}

\section{Conclusion}

In this paper, novel CMOS differential logic family with voltage bootstrap- 
ping is described. The proposed logic style provides higher switching speed than the conventional logic style at ultra-low supply voltage. The comparison results reveal that the proposed logic family provides up to $67 \%$ smaller power-delay product and occupies up to $17 \%$ less area than conventional ones, which indicates that the proposed logic family is well suited for applications in which speed performance must be maintained at scaled supply voltages.

\section{Acknowledgments}

This work was supported by Faculty research fund 2006, Sungkyunkwan University. Design tools were supported by IC Design Education Center (IDEC), KAIST. 